\title{
Evaluation of Mobile Application for the Management of Tuberculosis Patients in Tianjin During 2019-2020
}

\author{
Xiaorong $\mathrm{Li}$ \\ Xuewen Pang \\ Fan Zhang \\ Tianjin Center for Tuberculosis Control, \\ Tianjin, 30004I, People's Republic of \\ China
}

Purpose: Poor tuberculosis (TB) medication adherence increases the risk of treatment failure and development of drug-resistant TB, while universal implementation of directly observed therapy (DOT) is not feasible in China. EHealth technologies were reported to be promising patient-centered tools for improving adherence. However, only pilot studies have assessed patients' experiences, and the results were discrepant.

Patients and Methods: This prospective-cohort study was conducted among TB patients at the outpatient department from 3 March 2019 to 30 May 2020 in Tianjin, China. Data were downloaded from the Tuberculosis Doctor App and TB Information Management System (TBIMS) and merged them by the TBIMS notification number. Logistic regression analysis was used to analyze the factors associated with regular drug-intake. Odds ratios and 95\% confidence intervals were estimated with and without adjustment for age, gender, ethnicity and occupation.

Results: Revisit examination was more regularly and frequently in APP group than nonAPP group. In APP group, 33.28\% patients were regular drug-intake. The whole drug-intake rate was $84.84 \%$. Tuberculosis pleurisy (aOR: $0.42,95 \mathrm{CI} \%=0.26-0.69$ ) and retreated patients (aOR: $0.40,95 \mathrm{CI} \%=0.27-0.59$ ) were more likely to have poor medication compliance. Local residents tend to have better medication compliance (aOR: 1.80, 95CI\% $=1.16-2.79)$.

Conclusion: APP could improve TB patients' revisit examination adherence. Medication adherence was poor in tuberculosis pleuritis and retreated patients, while local residents tend to have better medication adherence. To make full use of the mobile application in TB patient management, more incentive measures should be adopted for patients and doctors, respectively.

Keywords: tuberculosis, mobile application, medication adherence

\section{Introduction}

Tuberculosis is a global public health problem. According to the estimates of World Health Organization (WHO), there were 9.96 million new cases of TB globally in 2019, with an incidence of 130/100,000 and 1.21 million deaths due to TB. ${ }^{1}$ China ranks the third in terms of TB burden in the world, accounting for $8.4 \%$ of the estimated new cases and 14\% of multiple drug resistant tuberculosis (MDR-TB) patients. The occurrence of MDR-TB is closely related to the patient's treatment adherence. Treatment adherence refers to the degree to which the patient's treatment-related behaviors (such as taking medication, regular review, et al) are
Correspondence: Fan Zhang

Tianjin Center for Tuberculosis Control, No. 124 Chifeng Road, Heping District, Tianjin, 30004I, People's Republic of China

Tel +86-|33|2|| 9095

Fax $+8622-24333673$

Email 13312119095@I26.com 
consistent with the doctor's prescription. ${ }^{2}$ Poor treatment adherence will lead to prolonged disease, recurrence, aggravation, and even the extensive spread of MDR-TB bacilli. ${ }^{3}$ This problem has become one of the obstacles to the control of the global TB epidemic. ${ }^{4}$ Therefore, improving adherence is still one of the effective public health measures to reduce the spread of TB and control the epidemic. ${ }^{5}$

Directly observed therapy (DOT) by health-care workers has been proven to ensure TB patients' medication adherence. ${ }^{6}$ However, due to the high cost of healthcare, level of economic development, availability of transportation, medical resources and privacy issues, it is difficult for many developing countries to fully implement DOT. ${ }^{7}$ The DOT Short-course strategy was implemented in 1992 in China and covered the entire country by $2005,{ }^{8}$ only $20 \%$ TB patients were supervised by health-care workers, others were on self-administered treatment or by family members. ${ }^{7,9}$ Studies showed that a lack of DOT, adverse effects, ${ }^{10}$ financial burdens often led to medication nonadherence in China. ${ }^{11,12}$ It was reported that more than $25 \%$ of TB patients missed at least one dose of anti-TB drugs, ${ }^{9,13}$ which was higher than the WHO recommendation of less than $10 \%{ }^{14}$ Thus, in the 2017 WHO's TB treatment guidelines, eHealth technology was suggested to be applied in the management of TB patients. ${ }^{15}$

As a new mobile communication and network technology in the healthcare industry, eHealth technology not only could provide humanized, convenient and highquality services for patients, but also improve the quality and efficiency of medical service. ${ }^{16-18}$ Short messaging service (SMS), video supervision, wirelessly observed therapy (WOT) and mobile application (APP) were reported to be applied in TB patients' management, ${ }^{19-21}$ although those measures contributed to improved compliance compared to DOT, no eHealth technology was widely promoted. ${ }^{22-25}$ APP supervision has shown its potential application due to its low cost, convenience and high acceptance. However, at present, only a small range of pilot studies have reported the effect of APP in the management of TB patients. ${ }^{26}$

In Tianjin, after early pilot applications and continuous improvement during 2015-2018, APP has been promoted and used in all districts since March 2019. To evaluate the effect of APP, we analyzed the data of TB patients who have completed the course of treatment in Tianjin, China.

\section{Materials and Methods}

\section{Study Design}

This is a prospective cohort study. The APP was applied in all districts since March 2019. After diagnosis, the patients were instructed to install the TB assistant APP by healthcare doctors of the community where they currently live. The TB assistant APP could remind the patient to take medicine and visit the hospital according to the set time. On the day of taking medicine, the information of taking medicine was uploaded when the patient signs in the TB assistant App. The APP will automatically send medication reminders to patients who did not sign-in at $10 \mathrm{AM}$ and 3 PM respectively. Sign in was equivalent to taking medicine. During the study, if the patients lost to followup, refused treatment, died, ruled out of TB, default due to adverse reactions, their data would be treated as invalid.

The doctor visited each patient within 72 hours after diagnosis, and contacted them at least once a month. The doctor evaluated the drug adherence, given advice about timely and regular drug intake. If a patient failed to sign in the App, the doctor should remind him/her through the TB doctor App; if a patient did not visit the hospital 3 days after the scheduled time, the doctor was required to call or visit patient to supervise him/her to visit the designated medical institution within $24 \mathrm{~h}$.

\section{Study Population}

The study population comprises a consecutive enrolled pulmonary TB patients diagnosed at clinic. Inclusion criteria were as follows: 1 ) $\geq 18$ years old; 2) Patients with no communication impairment (mental, visual, auditory, or speech); 3) Patients or family members agreed to download and use App after training: 4) Patients were able to persist in receiving treatment in Tianjin. In order to evaluate the review status, 2386 frequency matched (by age and gender) controls who refused to download APP were included as non-APP group.

All TB patients were given a standard 6-8 months short-course chemotherapy and managed as outpatients. The chemotherapy regimens include isoniazid, rifampin, ethambutol, and pyrazinamide for $2 \mathrm{mo}$, followed by isoniazid and rifampin for 4 or $6 \mathrm{mo}$; The therapy used every day dosing for the entire treatment course. At each monthly visit, patients went to hospital to get enough medications for a 1-mo period. DOT at a health-care facility often meant that a patient had to give up part of their working day to attend, ${ }^{27}$ it is difficult and unrealistic 
for most TB patients especially those who need to commute to work on time. So in Tianjin, DOT was conducted only in a minority of TB patients in rural areas, most TB patients taken medicine supervised by health-care doctors or self-administered treatment or supervised by family members. APP could serve as a reminder to overcome forgetfulness, especially those that are sent daily at set time, which could result into forming a habit of medication adherence by reminding patients to take their medicine. Health-care doctors could keep an eye on patients' daily medication and regular follow-up through the APP at any time.

This study was conducted in accordance with the Declaration of Helsinki.

\section{Introduction of APP}

App was developed by Beijing SINOVO POWER Technology Co., Ltd. and Beijing Chest Hospital. It consists of two parts: Tuberculosis Assistant App and Tuberculosis Doctor App. Tuberculosis Assistant App is available for TB patients, which could provide reminders of drug taking and review, Q\&A consultation, patient forum and communication with doctors. Tuberculosis Doctor App is the terminal of management doctor, including the mobile APP and the web system. Doctors can view medication feedback, respond to patients' questions, communicate with patients instantly, send messages and irregularly push health information, etc.

\section{Data Collection}

Data was downloaded from the Tuberculosis doctor App and TB Information Management System (TBIMS).

Observation indicators: (1) drug-intake rate: the drugintake rate of patients in different treatment months was calculated. The calculation formula was as follows: the monthly drug-intake rate $=$ the actual times of drugintake in the month/the times should be taken in the month $\times 100 \%$; the whole drug-intake rate $=$ the actual times of drug-intake during the whole course/the times should be taken during the whole course $\times 100 \%$; (2) Determination of regular drug-intake: The whole drugintake rate $\geq 90 \%$ was determined as regular drug-intake, otherwise as irregular drug-intake. (3) According to the diagnosis for pulmonary tuberculosis (WS 288-2017), TB patients must complete regular sputum smear/culture during treatment, and chest radiograph as reference, thus treatment adherence was defined as regularity of followup reexamination (sputum smear/culture at the end of the
2,5 , and $6 / 8$ month, chest radiograph at the end of the 2 and $6 / 8$ month as reference), thus the accumulate times of sputum smear, sputum culture above 3 times were regard as treatment adherence.

Case finding was classified as active treatment; while referral, follow-up, health examination and contact examination were classified as passive treatment.

\section{Statistical Analysis}

All analyses were performed using SPSS software (version 16.0; SPSS Inc., Chicago, IL, USA). Normality test was performed first for continuous variables, data that were not conforming to the normal distribution were presented as median (quartile), and non-parametric Kruskal-Wallis test was performed. Categorical data were evaluated by Chisquare test as appropriate. Univariate and multivariate logistic regression analysis was used to analyze the association between factors and regular drug-intake. All tests were two-sided and $P<0.05$ was considered statistically significant.

\section{Results}

\section{Patient Characteristics}

From 3 March 2019 to 30 May 2020, a total 1284 pulmonary TB patients were informed and agreed to download Tuberculosis Assistant App to finish their whole course of anti-tuberculosis treatment. Of those, 91 patients were excluded: 36 patients were loss follow-up, 23 patients subsequently refused to use the APP, 21 patients moved away, 6 patients were ruled out of TB and 5 patients died of other disease. Finally 1193 (92.91\%) patients met the enrollment criteria.

Among the 1193 study patients, 799 (66.97\%) were males and 394 (33.03\%) were females. The median age was 48 years old, the minimum age was 14 years old and the maximum was 95 years old. Three hundred and eighteen $(26.66 \%)$ patients were student and employee, 1052 (88.18\%) were local residents, 861 (72.17\%) were active case finding, 757(63.45\%) were diagnosed as negative and $349(29.25 \%)$ were positive, $1070(89.69 \%)$ were new TB patients. The basic characteristics were comparable between the APP group and non-APP group (Table 1).

\section{Comparison of Review Adherence}

In App group, the proportion of sputum smear in the 2, 5, 6 and 8 months were higher ( $\mathrm{P}<0.01$, respectively). Sputum culture in the $0,2,5,6$ and 8 month was more 
Table I Characteristics of APP Group and Non-APP Group

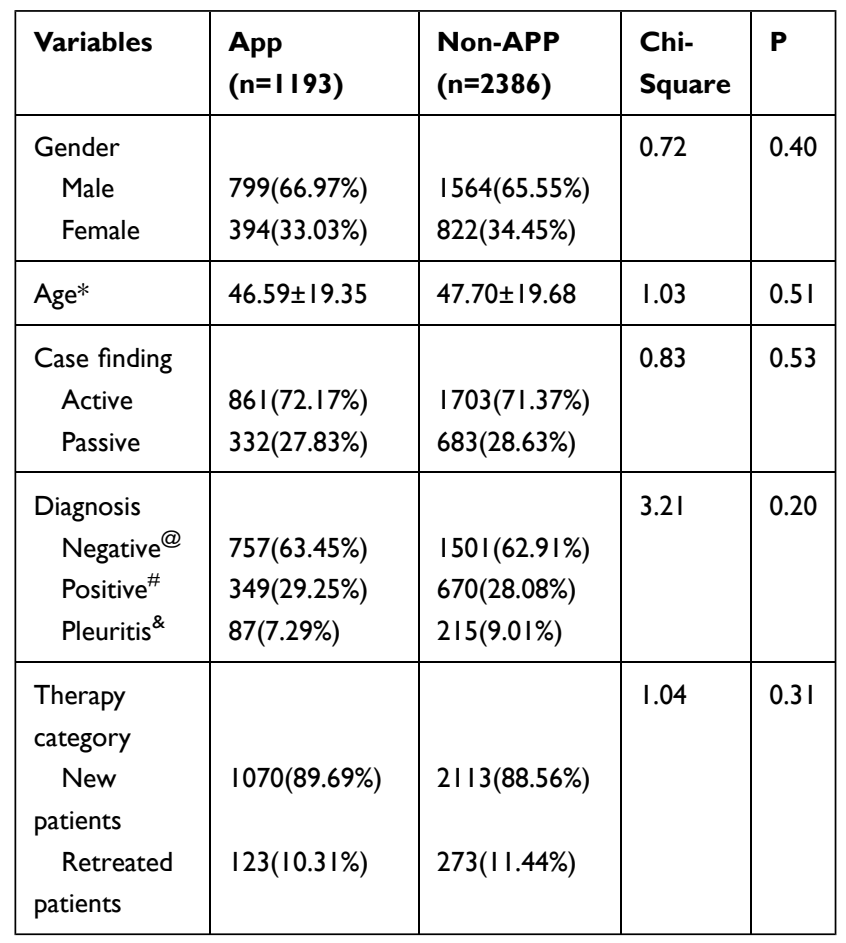

Notes: *Age is presented as mean \pm SD; ${ }^{\circledR}$ negative: the results of etiological examination of sputum was negative; " positive: the results of etiological examination of sputum was positive; \& pleuritic: the results of bacteriological examination of pleural fluid was positive. common ( $\mathrm{P}<0.05$, respectively). App group were more likely to have $\mathrm{X}$ ray in the $1,3,5$ and 6 months $(\mathrm{P}<0.05)$ (Table 2). The proportion of the accumulate times above 3 of sputum smear, sputum culture and $\mathrm{X}$ ray were more common in App group $(\mathrm{P}<0.05)$ (Table 3).

\section{Results of Drug-Intake Rate}

Three hundred and ninety-seven (33.28\%) patients were regular drug-intake. The times that all patients should take medicine were 238,815 , while the actual times recorded by APP were 202,602, the whole drug-intake rate was $84.84 \%$. The drug-intake rate at the first month was $51.84 \%$, and the drug-intake rate decreased with the month, this decreasing trend was significant $(P<0.01)$.

\section{Comparison of Patient Characteristics}

The demographic characteristics of TB patients are presented in Table 4. The proportion of 50 years old and above, migrants, new patients, and negative patients in irregular drug-intake group were all higher than those in the regular drug-intake group, the differences were statistically significant $(\mathrm{P}<0.05)$.

Table 2 Comparison of the Follow-Up Reexamination in Different Month

\begin{tabular}{|c|c|c|c|c|}
\hline Months & App $\left(n^{\#}=1193\right)$ & Non-APP $\left(n^{\#}=2386\right)$ & Chi-Square & $\mathbf{P}$ \\
\hline \multicolumn{5}{|c|}{ Sputum smear month } \\
\hline 0 month & II 76 (98.58\%) & $2347(98.37 \%)$ & 0.23 & 0.64 \\
\hline 2nd month & II54(96.73\%) & 1949(81.68\%) & 156.15 & $<0.0001$ \\
\hline 5th month & II 20(93.88\%) & $1516(63.54 \%)$ & 377.36 & $<0.0001$ \\
\hline 6th month & $1047(87.86 \%)$ & $|27|(53.27 \%)$ & $4 \mid 4.67$ & $<0.0001$ \\
\hline 8th month & $163(13.66 \%)$ & $178(7.46 \%)$ & 35.50 & $<0.0001$ \\
\hline \multicolumn{5}{|c|}{ Sputum culture month } \\
\hline 0 & $1102(92.37 \%)$ & $2064(86.50 \%)$ & 26.82 & $<0.0001$ \\
\hline 2nd month & $196(16.43 \%)$ & $311(13.03 \%)$ & 7.54 & 0.006 \\
\hline 5th month & $88(7.38 \%)$ & $99(4.15 \%)$ & 16.73 & $<0.0001$ \\
\hline 6th month & III(9.30\%) & $90(3.77 \%)$ & 45.92 & $<0.0001$ \\
\hline 8th month & $10(0.84 \%)$ & $8(0.34 \%)$ & 4.02 & 0.045 \\
\hline \multicolumn{5}{|l|}{$X$ ray month } \\
\hline 0 & $1192(99.92 \%)$ & 2384(99.92\%) & 0 & 1 \\
\hline Ist month & $38(3.19 \%)$ & $36(1.51 \%)$ & 11.04 & 0.0009 \\
\hline 2nd month & $169(14.17 \%)$ & $390(16.35 \%)$ & 2.87 & 0.09 \\
\hline 3th month & $38(3.19 \%)$ & $35(1.47 \%)$ & 11.75 & 0.0006 \\
\hline 4th month & $40(3.35 \%)$ & $55(2.31 \%)$ & 3.38 & 0.07 \\
\hline 5th month & $39(3.27 \%)$ & $48(2.01 \%)$ & 5.30 & 0.02 \\
\hline 6th month & I55(|2.99\%) & $228(9.56 \%)$ & 9.83 & 0.002 \\
\hline 7th month & $9(0.75 \%)$ & 14(0.59\%) & 0.35 & 0.55 \\
\hline 8th month & $17(1.42 \%)$ & $31(1.30 \%)$ & 0.10 & 0.76 \\
\hline
\end{tabular}

Note: ${ }^{*} \mathrm{n}$ means the number of patients who had sputum smear/ sputum culture/x-rays at different month. 
Table 3 Comparison of the Accumulate Times of Reexamination

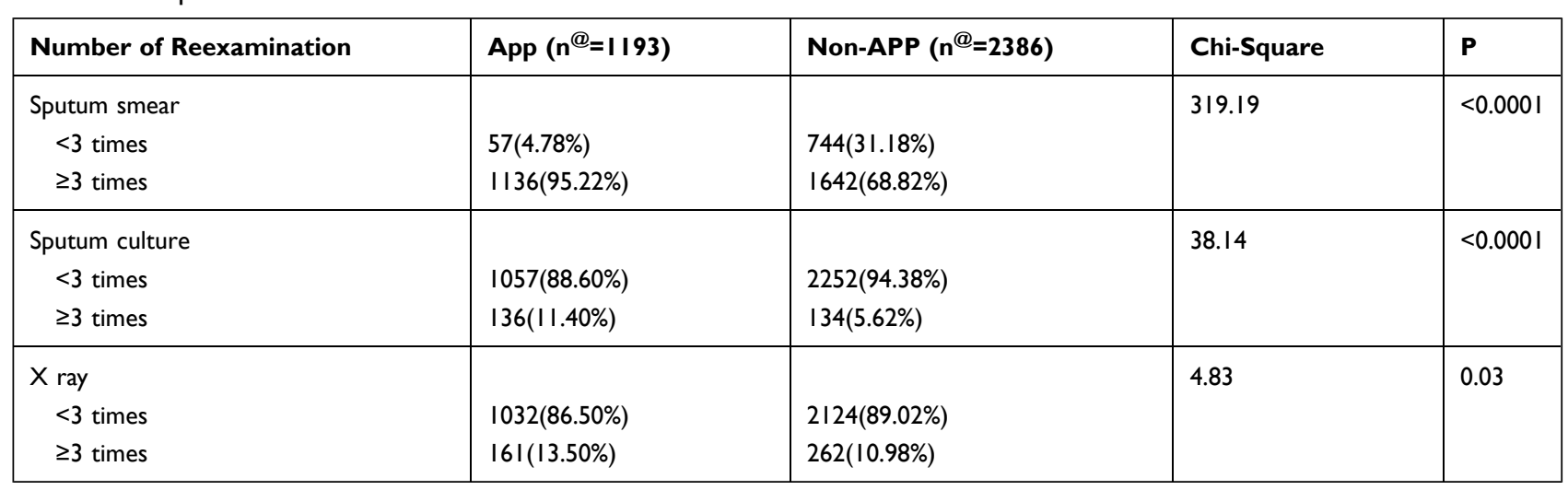

Note: ${ }^{\Theta_{n}}$ means the number of patients who had smear/culture/x-rays with different times.

Table 4 Characteristics of APP Group

\begin{tabular}{|c|c|c|c|c|}
\hline Characteristic & Irregular Drug-Intake & Regular Drug-Intake & Chi-Square & $\mathbf{P}$ \\
\hline $\begin{array}{l}\text { Gender } \\
\text { Male } \\
\text { Female }\end{array}$ & $\begin{array}{l}524(65.83 \%) \\
272(34.17 \%)\end{array}$ & $\begin{array}{l}275(69.27 \%) \\
122(30.73 \%)\end{array}$ & 1.42 & 0.24 \\
\hline $\begin{array}{l}\text { Age } \\
\qquad 50 \\
\quad \geq 50\end{array}$ & $\begin{array}{l}452(56.78 \%) \\
344(43.22 \%)\end{array}$ & $\begin{array}{l}200(50.38 \%) \\
197(49.62 \%)\end{array}$ & 4.39 & 0.04 \\
\hline $\begin{array}{l}\text { Ethnicity } \\
\qquad \text { Han } \\
\text { Others }\end{array}$ & $\begin{array}{l}784(98.49 \%) \\
12(1.51 \%)\end{array}$ & $\begin{array}{l}393(98.99 \%) \\
4(1.01 \%)\end{array}$ & 0.50 & 0.48 \\
\hline $\begin{array}{l}\text { Occupation } \\
\text { Student and employee } \\
\text { Farmers } \\
\text { Retired } \\
\text { Unemployed }\end{array}$ & $\begin{array}{l}220(27.64 \%) \\
191(23.99 \%) \\
90(11.31 \%) \\
295(37.06 \%)\end{array}$ & $\begin{array}{l}98(24.69 \%) \\
86(21.66 \%) \\
61(15.37 \%) \\
152(38.29 \%)\end{array}$ & 5.04 & 0.17 \\
\hline $\begin{array}{l}\text { Residence } \\
\text { Migrants } \\
\text { Local }\end{array}$ & $\begin{array}{l}\text { III(I3.94\%) } \\
685(86.06 \%)\end{array}$ & $\begin{array}{l}30(7.56 \%) \\
367(92.44 \%)\end{array}$ & 10.37 & 0.001 \\
\hline $\begin{array}{l}\text { Case finding } \\
\text { Active } \\
\text { Passive }\end{array}$ & $\begin{array}{l}56 I(70.48 \%) \\
235(29.52 \%)\end{array}$ & $\begin{array}{l}300(75.57 \%) \\
97(24.43 \%)\end{array}$ & 3.42 & 0.06 \\
\hline $\begin{array}{l}\text { Diagnosis } \\
\text { Negative } \\
\text { Positive } \\
\text { Pleuritis }\end{array}$ & $\begin{array}{l}252(31.66 \%) \\
500(62.81 \%) \\
44(5.53 \%)\end{array}$ & $\begin{array}{l}98(24.69 \%) \\
256(64.48 \%) \\
43(10.83 \%)\end{array}$ & 14.72 & 0.0006 \\
\hline $\begin{array}{l}\text { Therapy category } \\
\text { New patients } \\
\text { Retreated patients }\end{array}$ & $\begin{array}{l}740(92.96 \%) \\
56(7.04 \%)\end{array}$ & $\begin{array}{l}331(83.38 \%) \\
66(16.62 \%)\end{array}$ & 26.53 & $<0.0001$ \\
\hline
\end{tabular}




\section{Factors Associated with Irregular Drug-Intake}

As shown in Table 5, univariate logistic regression analysis showed that 50 years old and above were more likely to have poor adherence (OR: $0.77,95 \mathrm{CI} \%=0.61-0.98$ ). Retired TB patients demonstrated poor adherence (OR: $0.66,95 \mathrm{CI} \%=0.44-0.98)$. Compared with negative and positive TB patients, tuberculosis pleuritis were more likely to have poor adherence (OR: $0.40,95 \mathrm{CI} \%=0.25-$ 0.64). Retreated patients have poor medication compliance (OR: $0.38,95 \mathrm{CI} \%=0.26-0.56$ ). While local resident were related to good medication compliance (OR: 1.98, 95CI\% $=1.30-3.03$ ). After adjustment by age, gender, ethnicity and occupation, tuberculosis pleuritis (aOR: 0.42, 95CI\%
$=0.26-0.69)$ and retreated patients (aOR: 0.40, 95CI\% $=0.27-0.59$ ) were more likely to have poor medication compliance. Local resident tends to have better medication compliance (aOR: 1.80, 95CI\% $=1.16-2.79$ ).

\section{Discussion}

Effective management is the key to the success chemotherapy after the patient have been given an appropriate treatment plan. Although DOT has achieved great success in past decades, it was reported that in some areas the implementation rate of DOT was only $50 \%$ and $20 \%$ TB patients were self-administered therapy without any adherence support. $^{7}$ Therefore, how to make full use of high-tech methods to achieve accurate management of TB patients

Table 5 Factors Associated with Regular Drug-Intake for Pulmonary TB Patients

\begin{tabular}{|c|c|c|c|c|}
\hline \multirow[t]{2}{*}{ Variables } & \multicolumn{2}{|c|}{ Unadjusted OR } & \multicolumn{2}{|c|}{ Adjusted OR } \\
\hline & $O R^{\#}(95 \% \mathrm{CI})$ & P-value & aOR*(95\% Cl) & P-value \\
\hline \multicolumn{5}{|l|}{ Gender } \\
\hline Male & 1.00 & & 1.00 & \\
\hline Female & $0.17(0.90-1.52)$ & 0.23 & I.I0(0.84-I.42) & 0.53 \\
\hline \multicolumn{5}{|l|}{ Age } \\
\hline$<50$ & 1.00 & & 1.00 & \\
\hline$\geq 50$ & $0.77(0.6 \mathrm{I}-0.98)$ & 0.04 & $0.92(0.7 I-I .20)$ & 0.55 \\
\hline \multicolumn{5}{|l|}{ Ethnicity } \\
\hline Han & 1.00 & & 1.00 & \\
\hline Others & $0.66(0.2 I-2.08)$ & 0.48 & $0.69(0.22-2.23)$ & 0.54 \\
\hline \multicolumn{5}{|l|}{ Occupation } \\
\hline Student and employee & 1.00 & & 1.00 & \\
\hline Farmers & $0.99(0.70-1.40)$ & 0.24 & $1.05(0.7 I-1.53)$ & 0.49 \\
\hline Retired & $0.66(0.44-0.98)$ & 0.04 & $0.82(0.5 I-\mid .3 I)$ & 0.27 \\
\hline Unemployed & $0.87(0.64-1.18)$ & 0.98 & I.0I(0.72-I.4I) & 0.67 \\
\hline \multicolumn{5}{|l|}{ Residence } \\
\hline Migrants & 1.00 & & 1.00 & \\
\hline Local & $1.98(1.30-3.03)$ & 0.002 & $1.80(1.16-2.79)$ & 0.009 \\
\hline \multicolumn{5}{|l|}{ Diagnosis } \\
\hline Negative & 1.00 & & 1.00 & \\
\hline Positive & $0.76(0.58-1.00)$ & 0.20 & $0.8 \mathrm{I}(0.6 \mathrm{I}-\mathrm{I} .08)$ & 0.12 \\
\hline Pleuritis & $0.40(0.25-0.64)$ & 0.0005 & $0.42(0.26-0.69)$ & 0.0009 \\
\hline \multicolumn{5}{|l|}{ Therapy category } \\
\hline New patients & 1.00 & & 1.00 & \\
\hline Retreated patients & $0.38(0.26-0.56)$ & $<0.0001$ & $0.40(0.27-0.59)$ & $<0.0001$ \\
\hline \multicolumn{5}{|l|}{ Case finding } \\
\hline Active & 1.00 & & 1.00 & \\
\hline Passive & $1.30(0.98-1.7 \mid)$ & 0.06 & $1.14(0.85-1.53)$ & 0.37 \\
\hline
\end{tabular}

Abbreviations: ${ }^{\#} \mathrm{OR}$, odds ratio; $95 \% \mathrm{Cl}, 95 \%$ confidence interval; *aOR, adjusted $\mathrm{OR}$, odds ratio after adjustment by age, gender, ethnicity and occupation. 
is important to improve the level of TB prevention and treatment. At present, mobile medical APPs were applied in the management of chronic disease. Due to its relatively low cost and simple operation, APP was accessible and affordable even in resource-limited areas. However, its effect on improving the medication adherence of TB patients was discrepant currently. ${ }^{5,15,28,29}$

Community doctors were required to remind patients to return to hospital at least once a month. In this study, the sputum examination and $\mathrm{X}$ ray in APP group were more regularly and frequently than non-APP group. Currently the mobile phone has become a necessary article for daily use, it's both convenient and efficient for medical personnel to remind patients to take medicine and review on time through APP. In addition, mobile phone APP enables community doctors to timely know the treatment of patients and respond accordingly, ${ }^{30}$ which also promotes patients' medication and review adherence.

The whole drug-intake rate in our study was $84.84 \%$ (202602/238815), and only 33.28\% (397) patients were regular drug-intake. The drug-intake rate decreased with the month. At the first month, the drug-intake rate was the lowest of the whole therapy course; some patients often forgot to sign in on the APP after taking medicine. To improve the compliance of TB patients, $1 \mathrm{rmb} /$ day was given to patients in some districts since Aug 2019, the drug-intake rate of those TB patients who diagnosed after Aug 2019 was $98.32 \%$ at the first month, so the whole rate was underestimated. The drug-intake rate at the twice and third month increased compared with the drug-intake rate at the first month. However, from the fourth month, the drug-intake rate decreased. As to the possible reasons, on the one hand, after three months of standardized treatment, some patients stopped to take medicine when they felt relief of symptoms. On the other hand, doctors chosen to ignore the reminders of missing medication on the APP because of lacking sufficient financial incentives. ${ }^{31}$ In Tianjin, although according to the government strategy, a doctor can get $7 \mathrm{rmb}$ /day for supervising a patient to take medicine, however, for various reasons, the doctor actually got less than half of the money, or even none. Hence, improving the financial support of doctors and TB patients were beneficial for improving adherence.

Tuberculosis pleurisy patients tend to have poor medication adherence. Because tuberculosis is a respiratory infectious disease, the TB patients generally carry a psychological burden of being discriminated and lack of confidence in curing the disease. ${ }^{32}$ The attitudes of family members and friends were reported to influence the medication adherence. ${ }^{33}$ Patients with frequent supervision and mentally encouragement by family members, especially from spouses, were more likely to have better adherence. ${ }^{34}$ Compared with active TB, the therapy course of tuberculosis pleurisy was as long as 12 months, thus tuberculosis pleurisy patients often own more psychological burden, less social support and, therefore, poor medication adherence.

Retreated TB patients were showed to have poor adherence. Similar finding was reported in Wang et al study. ${ }^{35}$ Due to the long course of treatment, generally 6 to 12 months, some even as long as 18 months, and the adverse drug effects were more serious than those of new patients, retreated TB patients often had negative psychology and less confidence.

Our study noted that local residents were more likely to have better treatment adherence than migrants. Although free anti-tuberculosis drugs were provided by government, TB patients still need to pay extra fees for medical examinations and liver protection drugs. ${ }^{34}$ It was reported that treatment was sometimes interrupted due to financial burden of these additional cost. The reimbursement rate of medical insurance in China only refers to the policy reimbursement rate, and the actual reimbursement rate of patients is far lower than the policy rate. According to the investigations, the reimbursement rate of TB outpatient treatment in Tianjin was higher than the national average; ${ }^{36}$ in addition, migrants tended to have low income and low awareness of TB, they often stopped taking medicines once the symptoms improved. So more financial support was needed for the migrants to promote them to finish the therapy course.

\section{Limitations}

There were several limitations that need to be addressed. Firstly, since this study only included TB patients in Tianjin, due to the lack of multi-center data comparison, the extrapolation of the results were limited, but it can provide reliable scientific basis for the further development of community management strategies for TB patients in Tianjin. Secondly, due to some patients' failing to signin on APP after taking medicine, the data uploaded cannot truly reflect the patient's actual medication status. However, published data indicated that the sensitivity and specificity of $24 \mathrm{~h}$ medication monitor records as the evidence of TB drug adherence were respectively $99.52 \%$ and $95.45 \%$, there is a high correlation between 
medication monitors' records and rifampicin detected in urine, ${ }^{20}$ hence the records of APP were representative to some extent.

\section{Conclusions}

In this study, follow-up reexamination was more regularly and frequently in APP group than non-APP group. In APP group, medication adherence was poor in tuberculosis pleurisy and retreated patients, while local resident have better medication adherence. Non-adherence data should be noticed by doctors to provide more intensive supervision. In addition, financial support should be increased to improve the enthusiasm of doctors, so as to ensure the patients' adherence to complete the course of treatment.

\section{Abbreviations}

TB, Tuberculosis; MTB, Mycobacterium tuberculosis; APP, Application; DOT, Directly observed therapy; WHO, World Health Organization; SMS, Short messaging service; WOT, Wirelessly observed therapy; TBIMS, TB Information Management System.

\section{Ethics and Consent}

The study was approved by the review board of Tianjin Center for Tuberculosis Control and written informed consent was obtained from the patients.

\section{Acknowledgments}

We thank Shengyu Chen for clinical interpretation of the data.This study was supported by National Natural Science Foundation of China (No.81971660), Sichuan Science and Technology Program (No.2021YFH0004), and Tianjin Outstanding Youth Fund Project (No.20JCJQIC00230).

\section{Disclosure}

The authors report no conflicts of interest in this work.

\section{References}

1. World Health Organization. Global Tuberculosis Report 2020. Geneva, Switzerland: World Health Organization; 2020.

2. Coamb RB. Review of the Scientific Literature on the Prevalence, Consequences and Health Costs of Non Compliance Inappropriate Use of Prescription Medication in Canada: Health Promotion Research. Toronto: University of Toronto Press; 1995.

3. Karumbi J, Garner P. Directly observed therapy for treating tuberculosis. Cochrane Database Syst Rev. 2015;29:CD003343.

4. Van Den BJ, Lyimo RA, Boeree MJ, et al. Electronic monitoring of treatment adherence and validation of alternative adherence measures in tuberculosis patients: a Pilot Study. Bull World Health Organ. 2011;89(9):632-639. doi:10.2471/BLT.11.086462
5. Ma Y, Du J, Liu YH, et al. The role of mobile health on management of tuberculosis patients. Chin J Antituberc. 2016;38:527-530.

6. World Health Organization. Guidelines for Treatment of DrugSusceptible Tuberculosis and Patient Care 2017. Geneva, Switzerland: World Health Organization; 2017.

7. Hou WL, Song FJ, Zhang NX, et al. Implementation and community involvement in DOTS strategy: a systematic review of studies in China. Int J Tuberc Lung Dis. 2012;16(11):1433-1440. doi:10.5588/ ijtld. 12.0080

8. Wang L, Liu J, Chin DP. Progress in tuberculosis control and the evolving public-health system in China. Lancet. 2007;369 (9562):691-696. doi:10.1016/S0140-6736(07)60316-X

9. Wang L, Zhang H, Ruan Y, et al. Tuberculosis prevalence in China, 1990-2010; a longitudinal analysis of national survey data. Lancet. 2014;383(9934):2057-2064. doi:10.1016/S01406736(13)62639-2

10. Yao S, Huang WH, van den Hof S, et al. Treatment adherence among sputum smear-positive pulmonary tuberculosis patients in mountainous areas in China. BMC Health Serv Res. 2011;11(1):341. doi:10.1186/1472-6963-11-341

11. Chen S, Zhang H, Pan Y, et al. Are free anti-tuberculosis drugs enough? An Empirical Study from three cities in China. Infect Dis Poverty. 2015;4(1):47. doi:10.1186/s40249-015-0080-y

12. Lei X, Huang K, Liu Q, et al. Are tuberculosis patients adherent to prescribed treatments in China? Results of a prospective cohort study. Infect Dis Poverty. 2016;5(1):38. doi:10.1186/s40249-016-0134-9

13. Wei X, Hicks JP, Pasang P, et al. Protocol for a randomised controlled trial to evaluate the effectiveness of improving tuberculosis patients' treatment adherence via electronic monitors and an app versus usual care in Tibet. Trials. 2019;20(1):273. doi:10.1186/s13063-019-3364-x

14. Wolrd Health Organization. Global Tuberculosis Control: Surveillance, Planning, Financing: WHO Report 2005. Geneva: World Health Organization; 2005.

15. Falzon D, Timimi H, Kurosinski P, et al. Digital health for the end TB strategy: developing priority products and making them work. Eur Respir J. 2016;48(1):29-45. doi:10.1183/13993003.00424-2016

16. Haapala I, Barengo NC, Biggs S, et al. Weight loss by mobile phone: a 1-Year Effectiveness Study. Public Health Nutr. 2009;12 (12):2382-2391. doi:10.1017/S1368980009005230

17. Denkinger CM, Grenier J, Stratis AK, et al. Mobile health to improve tuberculosis care and control: a call worth making. Int J Tuberc Lung Dis. 2013;17(6):719-727. doi:10.5588/ijtld.12.0638

18. Free C, Phillips G, Galli L, et al. The effectiveness of mobile-health technology-based health behaviour change or disease management interventions for health care consumers: a systematic review. PLoS Med. 2013;10(1):e1001362. doi:10.1371/journal.pmed.1001362

19. Huan ST, Chen R, Liu XQ, et al. Operational feasibility of medication monitors in monitoring treatment adherence among TB patients. Chin J Antituberc. 2012;34:419-424.

20. Chuck C, Robinson E, Macaraig M, et al. Enhancing management of tuberculosis treatment with video directly observed therapy in New York City. Int J Tuberc Lung Dis. 2016;20(5):588-593. doi:10.5588/ijtld. 15.0738

21. Gashu KD, Gelaye KA, Mekonnen ZA, et al. Does phone messaging improves tuberculosis treatment success? A systematic review and meta-analysis. BMC Infect Dis. 2020;20(1):42. doi:10.1186/s12879020-4765-x

22. Bediang G, Stoll B, Elia N, et al. SMS reminders to improve adherence and cure of tuberculosis patients in Cameroon (TB-SMS Cameroon): a randomised controlled trial. BMC Public Health. 2018;18(1):583. doi:10.1186/s12889-018-5502-х

23. Nguyen TA, Pham MT, Nguyen TL, et al. Video directly observed therapy to support adherence with treatment for tuberculosis in Vietnam: a Prospective Cohort Study. Int $J$ Infect Dis. 2017;65:85-89. doi:10.1016/j.ijid.2017.09.029 
24. Gao L, Qian XJ, Huang SY, et al. The effect of traditional supervision and telephone supervision on treatment compliance of tuberculosis patients in county and township. Int $J$ Epidemiol Infect Dis. 2016;43:404-407.

25. Lewis JJ, Liu XQ, Zhang ZY, et al. Evaluation of a medication monitor-based treatment strategy for drug-sensitive tuberculosis patients in China: study protocol for a cluster randomised controlled trial. Trials. 2018;19(1):398. doi:10.1186/s13063-018-2650-3

26. Xie YT, Du J, Luo P, et al. Preliminary report of a mobile supervision application used for treatment management in patients with tuberculosis in Tongzhou District, Beijing in 2016. Chin J Antituberc. 2017;39:708-712.

27. Khan MA, Walley JD, Witter SN, et al. Tuberculosis patient adherence to direct observation: results of a Social Study in Pakistan. Health Policy Plan. 2005;20(6):354-365. doi:10.1093/heapol/czi047

28. Promthong K, Kittivoravitkul P, Oer-Areemitr N, et al. Tuberculosis treatment with smartphone medical application reminders a Randomised Control Study. Respirology. 2016;21:96.

29. Subbaraman R, de Mondesert L, Musiimenta A, et al. Digital adherence technologies for the management of tuberculosis therapy: mapping the landscape and research priorities. BMC Glob Health. 2018;3 (5):e001018. doi:10.1136/bmjgh-2018-001018

30. Chen X, Du L, Wu RH, et al. The effects of family, society and national policy support on treatment adherence among newly diagnosed tuberculosis patients: a Cross-Sectional Study. BMC Infect Dis. 2020;20(1):623. doi:10.1186/s12879-020-05354-3
31. Liu XQ, Lewis JJ, Zhang H, et al. Effectiveness of electronic reminders to improve medication adherence in tuberculosis patients: a cluster-randomised trial. PLoS Med. 2015;12(9):e1001876. doi:10.1371/journal.pmed.1001876

32. Theron G, Peter J, Zijenah L, et al. Psychological distress and its relationship with non-adherence to TB treatment: a Multicentre Study. BMC Infect Dis. 2015;15(1):253. doi:10.1186/s12879-0150964-2

33. Xu M, Markstrom U, Lyu J, et al. Detection of low adherence in rural tuberculosis patients in China: application of morisky medication adherence scale. Int J Environ Res Public Health. 2017;14(3):248. doi:10.3390/ijerph14030248

34. $\mathrm{Xu} \mathrm{WG}, \mathrm{Lu} \mathrm{W}$, Zhou Y, et al. Adherence to anti-tuberculosis treatment among pulmonary tuberculosis patients: a Qualitative and Quantitative Study. BMC Health Serv Res. 2009;9(1):169. doi:10.1186/1472-6963-9-169

35. Wang N, Zhang H, Zhou Y, et al. Using electronic medication monitoring to guide differential management of tuberculosis patients at the community level in China. BMC Infect Dis. 2019;19(1):844. doi:10.1186/s12879-019-4521-2

36. Xu CH, Li X, Liu XQ, et al. Analysis on outpatient medical expense reimbursement of tuberculosis in different medical insurance systems in China. Dis Surveil. 2018. Available from: http://kns.cnki.net/kcms/ detail/11.2928.R.20180622.0840.004.html. Accessed August 31, 2021 .
Patient Preference and Adherence

\section{Publish your work in this journal}

Patient Preference and Adherence is an international, peer-reviewed, open access journal that focusing on the growing importance of patient preference and adherence throughout the therapeutic continuum. Patient satisfaction, acceptability, quality of life, compliance, persistence and their role in developing new therapeutic modalities and compounds to optimize clinical outcomes for existing disease

\section{Dovepress}

states are major areas of interest for the journal. This journal has been accepted for indexing on PubMed Central. The manuscript management system is completely online and includes a very quick and fair peer-review system, which is all easy to use. Visit http:// www.dovepress.com/testimonials.php to read real quotes from published authors. 